\title{
ON THE EXISTENCE OF CONTACT FORMS
}

\author{
W. P. THURSTON AND H. E. WINKELNKEMPER
}

ABSTRACT. Using an old theorem of Alexander, we give a short and elementary proof that every closed, orientable 3-manifold has a contact form.

Introduction. Let $M^{m}(m=2 n+1)$ be a closed, smooth, orientable $m$ manifold; a contact form on $M$ is a smooth 1 -form $\omega$ such that $\omega \wedge(d \omega)^{n} \neq 0$ at each point. One knows [2] that the existence of a contact form on $M^{m}$ allows a reduction of the structure group of the tangent bundle of $M$ to $U(n)$ and hence, in particular, the odd-dimensional Stiefel-Whitney classes of $M$ have to vanish [4]. This, however, gives no information in dimension 3 and in [3] Chern ${ }^{1}$ asked: Does every closed, smooth, orientable 3-manifold admit a smooth contact form?

This question was first answered (in the affirmative) by Lutz [6] and Martinet [7]. They based their proof on the theorem that every closed, orientable 3-manifold can be obtained from $S^{3}$ by surgery, the same theorem used by Lickorish, Novikov and Zieschang to prove that every such manifold admits a codimension 1 foliation, i.e. a nonzero 1 -form $\eta$ on $M$ such that $\eta \wedge d \eta \equiv 0$; that is, in dimension 3, the exact opposite of a contact form.

It was observed by one of us (see also [5]) that the existence theorem for codimension 1 foliations on $M$ is an immediate consequence of the following

Theorem (Alexander [1]). Every closed, smooth, orientable 3-manifold $M^{3}$ is diffeomorphic to $W(h) \cup_{\mathrm{id}} \partial W \times D^{2}$, where $D^{2}$ is the 2-disc and $W$ is an orientable 2-manifold with boundary and $h: W \rightarrow W$ is a diffeomorphism which restricts to the identity on $\partial W ; W(h)$ denotes the mapping torus of $h$, i.e. the 3-manifold with boundary obtained from $W \times I$ by identifying $(x, 0)$ with $(h(x), 1), I=[0,1]$.

The object of this short note is to prove that the existence theorem for contact forms on 3-manifolds is also a more or less immediate and elementary consequence of Alexander's theorem.

Received by the editors July 23, 1974.

AMS (MOS) subject classifications (1970). Primary 53C15; Secondary 58A10, $58 \mathrm{~A} 30$.

${ }^{1}$ Also in Proc. U. S.-Japan Seminar in Differential Geometry (Kyoto, 1965), Nippon Hyoronsha, Tokyo, 1966; Question 1, p. 172. MR $35 \# 7268$. 
Construction of a contact form. We may assume that $h$ fixes a neighborhood of $\partial W$. Let $d \theta$ be a volume form for $\partial W$, and let $t$ be the collar parameter for a collar neighborhood $C$ of $\partial W$, in $W$, invariant by $h$.

Let $\alpha_{1}$ be any 1 -form which equals $(1+t) d \theta$ near $\partial W$. By Stokes' theorem, $\int_{W} d a_{1}=1$. If $\Omega$ is any volume form for $W$ which is $d t \wedge d \theta$ near $\partial W$, then, by de Rham's theorem, $\Omega-d \alpha_{1}=d \beta$, where $\beta$ is a 1-form which is zero near $\partial W$. Let $\alpha_{2}=a_{1}+\beta$. Then $\alpha_{2}$ satisfies (i) $d \alpha_{2}$ is a volume form, and (ii) $\alpha_{2}=(1+t) d \theta$ near $\partial W$.

The set of 1-forms satisfying (i) and (ii.) is convex. Hence, there is a 1 -form $\alpha$ on $W(b)$, such that $a$ restricted to any fiber, satisfies (i) and (ii). Since $d \theta$ and $t$ are defined in a natural way on $C \times S^{1}$, we may also specify that $\alpha=(1+t) d \theta$ in a neighborhood of $\partial W(h)$.

For a sufficient large constant $K$, it follows that $\omega=\alpha+K d \phi$ is a contact form on $W(b)$, where $d \phi$ is the pullback of a volume form for the base of the bundle $W(h)$ over $S^{1}$. In fact, $d \phi \wedge d \alpha$ is a nonsingular 3-form on $W(b)$, so $\omega \wedge d \omega=K(d \phi \wedge d \alpha)+\alpha \wedge d \alpha$ will also be nonsingular when $K$ is large.

We will now extend $\omega$ over $\partial W(h) \times D^{2}$. We will use coordinates $(\theta, r$, $\phi)$, where $(r, \phi)$ are polar coordinates for $D^{2}$. We may consider $C \times S^{1}$ to be $\partial W(h) \times\left(D_{2}^{2}-D^{2}\right)$, where $D_{2}^{2}$ is the disk of radius two, and the present coordinates are related to the old coordinates by $\phi=\phi, \theta=\theta, r=1+t$, so in the new coordinates, $\omega=r d \theta+K d \phi$ in $\partial W(h) \times\left(D_{2}^{2}-D^{2}\right)$. The form $-d \theta$ $+r^{2} d \phi$ is a smooth contact form near $r=0$ : this may be checked by converting to cartesian coordinates for $D^{2}$. Consider a 1 -form $\omega$ which may be written $\omega=f_{1}(r) d \theta+f_{2}(r) d \phi$. $\omega$ is a contact form away from $r=0$, iff

$$
\omega^{\wedge} d \omega=\left|\begin{array}{ll}
f_{1}(r) & f_{1}^{\prime}(r) \\
f_{2}(r) & f_{2}^{\prime}(r)
\end{array}\right| d \theta^{\wedge} d r^{\wedge} d \phi \neq 0 .
$$

That is, $\omega$ is a contact form iff the position vector and the tangent vector of the curve $\left(f_{1}(r), f_{2}(r)\right)$ in $\mathbf{R}^{2}$ are linearly independent, for $r \neq 0$. It is now clear that there is a curve $\left(f_{1}(r), f_{2}(r)\right)[0<r \leq 2]$ such that $\left(f_{1}(r), f_{2}(r)\right)=$ $\left(-1, r^{2}\right)$ near $r=0,\left(f_{1}(r), f_{2}(r)\right)=(r, K)$ for $1 \leq r \leq 2$, and such that $\omega=$ $f_{1}(r) d \theta+f_{2}(r) d \phi$ is a contact form.

This completes the construction.

\section{REFERENCES}

1. J. W. Alexander, A lemma on systems of knotted curves, Proc. Nat. Acad. Sci. U.S.A. 9 (1923), 93-95.

2. S. S. Chern, Pseudo-groupes continus infinies, Géométrie Différentielle (Colloq. Internat. Centre National de la Recherche Scientifique, Strasbourg, 1953), Centre National de la Recherche Scientifique, Paris, 1953, pp. 119-136. MR 16, 112.

3. S. S. Chern, The geometry of G-structures, Bull. Amer. Math. Soc. 72 (1966), 167-219. MR $33 \# 661$. 
4. J. W. Gray, Some global properties of contact structures, Ann. of Math. (2) 69 (1959), 421-450. MR 22 \#3016.

5. H. B. Lawson, Jr., Foliations, Bull. Amer. Math. Soc. 80 (1974), 369-418.

6. R. Lutz, Sur quelques propriétés des formes différentielles en dimension trois, Thèse, Strasbourg, 1971.

7. J. Martinet, Formes de contact sur les variétés de dimension 3, Proc. Liverpool Singularities Sympos. II, Lecture Notes in Math., vol. 209, Springer-Verlag, Berlin and New York, 1971, 142-163.

DEPARTMENT OF MATHEMATICS, PRINCETON UNIVERSITY, PRINCETON, NEW JERSEY 08540

DEPARTMENT OF MATHEMATICS, UNIVERSITY OF MARYLAND, COLLEGE PARK, MARY LAND 20742 